[Submitted to the Third Trieste IUPAP Semiconductor Symposium, "High Excitation and Short Pulse Phenomena," July 2-ī, 1984, Trieste, Italy]

By acceptance of this article, the publisher or recipient acknowledges the U.S. Government's right to retain a nonexclusive, royalty.frga license in and to any copyright covering the article.

\author{
Prepared by the \\ Solid State Division \\ Oak Ridge National Laboratory \\ 0ak Ridge, Tennessee 37831 \\ operated by \\ Martin Marietta Energy Systems, Inc. \\ for the \\ U.S. DEPARTMENT OF ENERGY \\ under Contract No. DE-AC05-840R21400 \\ June 1984
}




\title{
STUDIES OF PULSED LASER MELTING AND RAPID SOLIDIFICATION \\ USING AMORPHOUS SILICON*
}

\author{
D. H. Lowndes and R. F. Wood \\ Solid State Division, Oak Ridge National Laboratory \\ Oak Ridge, Tennessee 37831
}

\section{ABSTRACT}

Pulsed-laser melting of ion implantation-amorplized silicon layers, and the subsequent solidification of undercooled liquid silicon, have been studied experimentally and theoretically. Measurements of the time of the onset of melting of amorphous silicon layers, during an incident laser pulse, have been combined with measurements of the duration of melting, and with modified melting model calculations to demonstrate that the thermal conductivity, $K_{a}$, of amorphous silicon is very low $\left(K_{a} \cong 0.02 \mathrm{~W} / \mathrm{cm}-K\right)$. $K_{a}$ is also found to be the dominant parameter determining the dynamical response of amorphous silicon to pulsed laser radiation; the latent heat of fusion and melting temperature of amorphous silicon are relatively unimportant. Transmission electron microscopy indicates that bulk (volume) nucleation occurs directly from the highly undercooled liquid silicon that can be prepared by pulsed laser melting of amorphous silicon layers at low laser energy densities. A modified thermal melting model has been constructed to simulate this effect and is presented. Nucleation of crystalline silicon apparently occurs at a nucleation temperature, $T_{n}$, that is higher than the temperature, $T_{a}$, of the liquid-to-amorphous phase transition. The model calculations demonstrate that the release of latent heat by bulk nucleation occurring during the melt-in process is essential to obtaining agreement with experimentally observed depths of melting. 
These calculations also show that this release of latent heat accompanying bulk nucleation can result in the existence of buried molten layers of silicon in the interior of the sample after the surface has solidified. It is pointed out that the occurrence of bulk nucleation implies that the liquid-to-amorphous phase transition (produced using picosecond or ultraviolet nanosecond laser pulses) cannot be explained by purely thermodynamic considerations.

\section{Introduction}

In this paper we summarize the results of recent time-resolyed reflectivity measurements $[1-3]$ and model calculations [1-4] which, together with post-irradiation tränsmission electron microscopy (TEM) studies $[3,5]$, have allowed us to study both the transformation of amorphous (a) silicon to a highty undercooled liquid (\&) phase and the subsequent rapid solidification process. Our work was motivated by the following considerations.

On the one hand, there have been a number of recent theoretical and experimental attempts [6-12] (the experiments using both pulsed and continuous heating) to determine thermodynamic properties of a-Si, such as its melting temperature, $T_{a}$, and latent heat of fusion, $L_{a}$. These efforts have resulted in estimates for the difference between $T_{a}$ and $T_{c}$, the melting temperature of crystalline (c) Si, ranging from no experimentally observable difference $\left(T_{a} * T_{c}\right)[6,7]$ to $\left(T_{c}-T_{a}\right) \sim 500 \mathrm{~K}[11]$. The two most recent studies, by Donovan et al. [8] and by 01 son and co-workers $[6,7]$, used the results of continuous heating of a-Si in the solid phase to estimate properties such as $\mathrm{T}_{\mathbf{a}}$ and $\mathrm{L}_{\mathbf{a}}$; both studies resulted in estimates for $T_{a}$ near the middle of this range [i.e., $\left(T_{c}-T_{a}\right) \sim 200-300 \mathrm{~K}$, 
though neither directly observed an a $\rightarrow \ell$ transformation. We have been interested in whether an alternative approach, using time-resolved measurements and related model calculations, could provide additional information about the thermodynamic parameters or thermophysical properties of a-Si (such as its thermal conductivity, $k_{a}$ ), and help to illuminate the reasons for the wide range of values obtained in previous studies.

Our time-resolved measurements employ a newly developed technique $[1,2]$ that allows us to determine the time, $t_{m}$, of the onset of melting of ionimplanted a Si, during an incident laser pulse, as well as conventional measuraments of the duration, $\tau_{S}$, of the high reflectivity phase (HRP) associated with surface melting, both as functions of the laser energy density, $E_{\ell}$. One of the principal results of the work reported here is a demonstration of the dominant role played by the low thermal conductivity, $\mathrm{K}_{\mathrm{a}}$, of $\mathrm{a}-\mathrm{Si}$, relative to that of $\mathrm{C}-\mathrm{S} i$, in determining both the melting threshold value of $E_{\ell}$ for a-Si layers of varying thicknesses, and the subsequent solidification behavior of partially molten layers of a-Si, at higher $E_{\ell}$. The $t_{m}$ measurements provide a particularly powerful constraint in fixing the value of $K_{a}$. We conclude that the dynamics of pulsed laser melting and resolidification are relatively insensitive to the values of the melting temperature, $T_{a}$, and the latent heat of fusion, $L_{a}$, of $a-S i$. This result seems particularly significant in view of the conflicting conclusions regarding $T_{a}$ in earlier measurements.

The second factor motivating our work was the realization that, since a-Si apparently does melt at $\mathrm{T}_{a} \ll \mathrm{T}_{C}$, then pulsed laser melting of a-Si, using laser pulses of low energy density, $E_{\ell}$, provides a means for preparing a melt of $1-S i$ that is dramatically undercooled, relative to its 
normal freezing point. Relatively well-characterized a-Si (free of voids and gaseous impurities) can be prepared by ion implantation into a c-Si substrate. By melting only partially through such an a-Si layer, the undercooled $\mathrm{I}-\mathrm{Si}$ remains isolated from the $\mathrm{C}-\mathrm{Si}$ beneath and the mechanism for resolidification under conditions of large undercooling can then be studied. Does solidification proceed from the 1-a boundary (as in liquid phase epitaxy from a crystalline substrate)? Or, will some form of bulk (volume) nucleation and growth be observed in the absence of a crystalline template?

Cross-sectional TEM micrographs $[3,5]$ provide important evfdence that bulk nucleation does occur under these conditions. Furthermore, thermal melting model calculations of melt depth and melt duration can be accurately "calibrated," by comparison with time-resolved reflectivity measurements of the duration of the high reflectivity phase (surface melt duration). These model calculations then can be used to infer temperature profiles in the undercooled 1-Si, and the nucleation behavior of undercooled 1-Si can be determined by comparison with the solidification profiles observed in TEM and by using the theory of homogeneous nucleation $[3,4]$. Using this approach, we have been able to estimate a nucleation temperature, $T_{n}$, where $T_{a}<T_{n}<T_{c}$. Thus, bulk nucleation apparently occurs in undercooled $1-\mathrm{Si}$ at temperatures above that of the $1 \rightarrow$ a transition [4]. This conclusion is important since the conventional thermodynamic interpretation of the laser-induced conversion of C-Si to a-Si apparently requires undercooling to $T_{a}$ for this conversion to occur [13]. Our work, revealing bulk nucleation at $T_{n}>T_{a}$, seems to imply that the 
$1 \rightarrow$ a transition cannot be explained by purely thermodynamic considerations and that nonequilibrium and kinetic effects must play an important role. Thus, the combination of techniques described in this paper provides a powerful method for studying the dynamics of rapid solidification under conditions of large undercooling of the melt.

\section{Time-Resolved Measurements}

A frequency-doubled $\mathrm{Nd}$ laser followed by four dichroic mirrors was used to provide a harmonically pure $532-\mathrm{nm}$ beam with $(18 \pm 1)$-nsec pulse duration. The laser was operated at fixed amplifier gain to avoid slight gain-dependent changes of second-harmonic pulse width; $E_{\ell}$ was varied with lenses; a beam splitter was used for in-situ monitoring of the energy of every laser shot; a separate vacuum planar photodiode and storage oscilloscope were used to monitor the temporal shape of each pulse. Only laser pulse shapes that were temporally nearly Gaussian were used in the analyses. A cw HeNe probe laser beam, incident at $10^{\circ}$ to the sample normal and focused to a 35-um-diam (1/e) spot, a silicon avalanche photodiode detector, and a storage oscilloscope (time constant $\sim 1$ nsec) were used to monitor the time-resolved reflectivity, $R$. The a-Si layers were produced by implantation of $40-\mathrm{keV}$ Si ions at a dose of $6 \times 10^{15} / \mathrm{cm}^{2}$ into (100) $\mathrm{n}$ type silicon of $1-5 \Omega \mathrm{cm}$ resistivity; transmission electron microscopy (TEM) showed the amorphous layer to be slightly greater than $100 \mathrm{~nm}$ thick.

Figure 1 shows results for $t_{m}$ as a function of $E_{l}[2,3]$; the data for C-Si were taken from Ref. 1. These data were obtained by simultaneously monitoring the time evolution of the $R$ signal and of the incident 532-nm laser pulse and determining $t_{m}$ during the incident laser pulse. To 
further reduce errors due to slight variations in pulse shape, the zero of time for these measurements was taken to be the center of the incident laser pulse; positive times correspond to melting before the peak of the pulse. The experimental $t_{m}$ is defined as the time when the HRP is first reached. As is expected intuitively, c-Si melts only during the latter half of the laser pulse at low $E_{\ell}$, with $t_{m}$ moving to the front of the laser pulse with increasing $E_{\ell}$ (Fig. 1). $t_{m}$ for a-Si exhibits a similar qualitative dependence on $\mathrm{E}_{\ell}$ but a-Si, in contrast to $\mathrm{C}-\mathrm{Si}$, is never observed to melt as late as the peak of the laser pulse. In fact, for $E_{\ell}$ $>0.6 \mathrm{~J} / \mathrm{cm}^{2}$, a-Si melts within the first few nanoseconds of the laser pulse, some 12-13 nsec prior to its peak.

Figure 2 shows the results of our measurements of $\tau_{S}$ vs $E_{\ell}$ for a-Si $[2,3]$. Also shown for comparison are results of recent $\tau_{s}$ measurements, under nearly identical conditions, for C-Si [1]. The most apparent differences between the two sets of results are that (1) a-Si melts at a significantly lower value of $E_{\ell}\left(\sim 0.15 \mathrm{~J} / \mathrm{cm}^{2}\right)$ than does $c-S i\left(E_{\ell}=0.35\right.$ $\mathrm{J} / \mathrm{cm}^{2}$ ) and (2) $\tau_{s}$ for a-Si displays a quasiplateau from $\sim 0.25$ to $\sim 0.4 \mathrm{~J} / \mathrm{cm}^{2}$.

\section{Thermal Conductivity of Amorphous Silicon}

For C-Si we recently found [1] that near the melting threshold, model calculations of $\tau_{s}$ and $t_{m}$ are quite sensitive to the values used for the optical and thermal properties of $\mathrm{C}-\mathrm{Si}$. This result provides confidence that the process may be inverted, with the observed behavior of $t_{m} v s E_{\ell}$ being used as a constraint in order to infer thermophysical properties of interest for $\mathrm{a}-\mathrm{Si}[2,3]$. In melting model calculations for $a-5 i$, we used a reflectivity $R_{a}=0.42$ and absorption coefficient $\alpha=5 \times 10^{5} \mathrm{~cm}^{-1}$, 
independent of $T$ in the solid [14]. When the first cell $(10 \mathrm{~nm})$ in the finite difference calculations melts, $\alpha$ is increased to $10^{6} \mathrm{~cm}^{-1}$ and $\mathrm{R}$ to 0.70. Exploratory calculations were made with many different pairs of values of $T_{a}$ and $L_{a}$ (see discussion below) but our baseline calculations assumed $\mathrm{L}_{a}=1320 \mathrm{~J} / \mathrm{gm}$ and $\mathrm{T}_{a}=1150^{\circ} \mathrm{C}$, in close agreement with the estimates of Donovan et al. [8]. The thermal conductivity, $K_{C}$, of $c-S i$ was taken from Glassbrenner and Slack [15] while $K_{\ell}$ for $\ell-S i$ was related to the electrical conductivity in $\ell-S i$ by the Wiecomann-Franz law [1].

We at first assumed a temperature-independent $k_{a}$ for a-Si equal to the high temperature $c-S i$ value of $\simeq 0.25 \mathrm{~W} / \mathrm{cm}-K[15]$, but the $t_{m}$ measurements showed that this could not be correct. $K_{a}$ was then successively halved until it was in the range $0.01-0.03 \mathrm{~W} / \mathrm{cm}-K$, when satisfactory agreement with experiment was finally obtained [2]. The value $k_{a}=0.02$ $W / \mathrm{cm}-K$ was used subsequently in the calculations, unless otherwise stated; this value represents an average over the expected weak $T$-dependence of $K_{a}$ above room temperature.

The solid curves lying close to the measured data in Fig. 1 summarize the agreement that can be obtained between experiment and calculations of $t_{m}$ vs $E_{\ell}$ for $a-$ and $\mathrm{C}-\mathrm{S} \mathrm{S}$. The dashed curve in the " $\mathrm{C}-\mathrm{S} i$ " region $\mathrm{illustra-}$ tes the results of calculations in which we used $\mathrm{T}_{\mathrm{a}}=1150^{\circ} \mathrm{C}$, and $\mathrm{L}_{\mathrm{a}}=$ $1320 \mathrm{~J} / \mathrm{gm}$ but took $\mathrm{K}_{\mathrm{a}}=\mathrm{K}_{\mathrm{c}}$. It is clear that the reduction of melting temperature and latent heat from crystalline values $\left(L_{C}=1799 \mathrm{~J} / \mathrm{gm}\right.$ and $T_{C}$ $=1410^{\circ} \mathrm{C}$ ) has little effect on $t_{m}\left(E_{\ell}\right)$ : It is the value of $k_{c}$ that defines the dynamical melting behavior as being that of C-Si. The dashed curve in the "a-Si" region of Fig. 1 reinforces this conclusion, since it 
was obtained by putting $T_{a}=T_{c}=1410^{\circ} \mathrm{C}, L_{a}=1320 \mathrm{~J} / \mathrm{gm}$ but keeping $\mathrm{K}_{\mathrm{a}}=$ $0.02 \mathrm{~W} / \mathrm{cm}-\mathrm{K}$. These results provide convincing evidence for the dominant role of $k_{a}$ in determining $t_{m}[2]$.

The value $k_{a} \simeq 0.02 \mathrm{~W} / \mathrm{cm}-K$ determined here for self-implantationamorphized a-Si is quite reasonable. A lattice thermal conductivity calculation, using a Dooye phonon spectrum for $\mathrm{a}-\mathrm{Si}$, gives $\mathrm{K}_{\mathrm{a}}(300 \mathrm{~K})=$ $0.011 \mathrm{~W} / \mathrm{cm}-\mathrm{K}$, while Goldsmid et al. [16] recently obtained $\mathrm{K}_{\mathrm{a}}(293 \mathrm{~K})=$ $0.026 \mathrm{~W} / \mathrm{cm}-\mathrm{K}$ for a $1.15-\mu m$ thick deposited film of a-Si on sapphire. Webber et al. [17] have also estimated that $K_{a} \sim 10^{-2} \mathrm{~W} / \mathrm{cm}-K$ via computer modeling of pulsed laser melting of ion-implanted a-Si layers. .

\section{Transmission Electron Microscopy: Evidence for Bulk Nucleation}

Cross-sectional TEM micrographs have been published $[3,5]$ illustrating the solidification morphologies that are observed following pulsed ruby laser (693 nm wavelength, 12-nsec FWHM pulse duration) irradiation of a $500-\mathrm{nm}-$ thick a-Si layer produced by $30_{S j}{ }^{+}$ion implantation of (100) c-Si at liquid nitrogen temperatures. The published TEM results $[3,5]$ are typical of the solidification morphologies that were observed in 100 to 500-nm-thick a-Si layers, using both pulsed Nd:YAG and pulsed ruby lasers and will simply be summarized here for the case of a 500-nm-thick a-Si layer.

No microstructural change was observed compared to the as-implanted state at $E_{\ell}=0.1 \mathrm{~J} / \mathrm{cm}^{2}$. However, for $E_{\ell}$ slightly less than $0.2 \mathrm{~J} / \mathrm{cm}^{2}$, the surface melts as indicated by a jump in reflectivity. At $E_{\ell}=0.2$ $\mathrm{J} / \mathrm{cm}^{2}$, the top $183 \mathrm{~nm}$ of a-Si was transformed into a very fine-grained polycrystalline (FG) region upon solidification, with average grain size 
$\sim 10 \mathrm{~nm}$. At $0.3 \mathrm{~J} / \mathrm{cm}^{2}$, two distinct regions were formed: a surface region (80 nm deep) containing large columnar polycrystalline grains (known. henceforth as the $L G$ region) and a deeper layer (233 nm thick) containing FG material similar to that formed alone at $0.2 \mathrm{~J} / \mathrm{cm}^{2}$. With further increase of $E_{\ell}$, the thickness of the $L G$ region grows linearly with $E_{\ell}$, while the thickness of the FG region reaches a maximum of about $240 \mathrm{~nm}$ at $E_{\ell}=0.5 \mathrm{~J} / \mathrm{cm}^{2}$ and then decreases with further increase of $E_{\ell}$. The LG region disappears completely only when complete epitaxy occurs, with melting through of the a-c interface at high $E_{\ell}$. Thus, for lower $E_{\ell}$ (insufficient to melt through to the a-c interface), the sequenee of materials observed is LG $p-S i$ near the surface, followed in order of increasing depth by $\mathrm{FG} p-S i$, the remaining unmelted $a-S i$, and the $c-S i$ substrate; for sufficiently low $E_{\ell}$ the $L G$ region is absent.

A microdiffraction pattern taken from the FG region showed that diffraction rings characteristic of amorphous $\mathrm{Si}$ were not observed; thus, the FG p-Si region marks an abrupt boundary with the a-ji beneath. The possibility that the $F G$ region results from solid phase nucleation and growth (SPNG) can be ruled out because (1) there is insufficient time for SPNG to result in $\sim 10 \mathrm{~nm}$ crystallites (the minimum time required even at the melting point of $\mathrm{C}-\mathrm{Si}$ is $\sim \mathrm{I} \mu \mathrm{sec}$ ) [6]; and (2) a sharp boundary is observed between the FG and underlyins amorphous regions, rather than the diffuse boundary expected for SPNG [5]. The grains of FG P-Si were randomly oriented and equiaxed, indicating that there is no preferred direction for crystal growth during formation of the FG $\mathrm{p}-\mathrm{Si}$ region, and certainly no microscopic evidence for the existence of a planar solidification front. This behavior is strongly suggestive of bulk nucleation 
occurring in highly undercooled $\ell-S i$. Insight into its origins is obtained from the caiculations described next.

\section{Model Calculations: Solidification of Highly Undercooled e-Si}

To our knowledge, a formulation of the heat flow problem incorporating bulk nucleation, large undercoolings, and a pulsed heat source is not yet available. Nevertheless, we have found that finite-difference calculations [18] can be modified to simulate nucleation effects under such conditions. Calculations of the type discussed in Refs. 1 and 18 should be adequate for determining the onset of melting, $t_{m}$, but it is not clear that they can be modified to describe subsequent solidification from a highly undercooled mel:. More specifically, such calculations in the past have generally assumed that the material gives up latent heat at the same temperature at which it is absorbed. In the case of an a-Si. layer with $T_{a}=1150^{\circ} \mathrm{C}$, the material would have to $\mathrm{cool}$ to $1150^{\circ} \mathrm{C}$ before solid-, ifying. This is an unnecessarily restrictive condition which, if valid, would seem to imply from thermodynamic considerations that the material could only resolidify as a-Si. However, the presence in typical transmission electron micrographs of randomly oriented FG p-Si suggests that the $F G$ material marks a region in which homogeneous bulk nucleation has occurred at some $T<T_{n}$, the nucleation temperature, lying between $T_{a}$ and $T_{C}$. The volumetric release of latent heat on bulk nucleation of the $c$ phase is accompanied by either a very rapid solidification or the creation of a "slush" zone $[18,19]$ from which solidification subsequently proceeds at a rapid rate; during this time it may be that no well-defined melt front exists. Nucleation does not occur (or occurs very slowly) in the 
molten Si with $T>T_{n}$, until a melt front is reestablished at the interface with the iine-grained material.

With the foregoing in mind, the calculations were modified from those of Ref. I and 18 to include not only the presence of the a-Si layer but also a simulation of the effects of bulk nucleation. We emphasize that only one physical and mathematical model of an a-Si layer on a c-Si substrate was used in all of the calculations discussed in this paper. We discussed the results for $t_{m}$ before considering the need to include undercooling and bulk nucleation in the model only because these two effects are not expected to have any significant influence on $t_{m}$, whereas they may greatly influence $\tau_{s}$. While the melt front is still advancing into the a-Si layer, $L_{a}, T_{a}$, and $K_{a}, K_{c}$, or $K_{\ell}$ are used depending on the phase of the material in a given finite-difference cell. The latent heat is always switched from $L_{a}$ to $L_{c}$ as soon as an a-Si cell has melted; this is the simplest assumption consistent with the transmission electron microscopy observations. If the melt front does not penetrate to the a-c interface, a layer of a-Si with its low thermal conductivity remains. The $\ell-S i$ in a given cell is allowed to solidify at the temperature of that cell, provided that this $T$ is less than $T_{n}$ and that the melt front has begun to recede from its point of maximum penetration. For those molten cells in which $T$ is initially $>T_{n}$ but $\left\langle T_{c}\right.$, crystallization cannot occur until $T$ drops below $T_{n}$ or until the rapidly growing region of fine-grained $S i$ from an adjacent cell can provide nucleation sites for the liquid with $T>T_{n}$. When the melt front initially contacts the a-c interface, the temperature of the interface, $T_{a c}$, will be $T_{a}$. The melt front cannot penetrate 
further until $T_{a c}$ rises to $T_{c}$ and enough latent heat is supplied to begin to melt the $C-S i$ region. If $E_{\ell}$ is not great enough for $T_{a c}$ to reach $T_{C}$, crystallization will occur at temperatures between $T_{a}$ and $T_{C} \cdot A$ thin layer of fine-grained Si may be formed if $T_{a c}<T_{n}$ but otherwise only large-grained $c-S i$ will be formed. Finally, when $T_{a c} \geq T_{c}$, epitaxial regrowth of single-crystal $\mathrm{Si}$ from the $\mathrm{c}$-Si substrate can proceed just as it would for an entirely c-Si sample.

The results of calculations of $\tau_{S}$ for a 100-nm-thick a-Si layer on a $\mathrm{C}-\mathrm{Si}$ substrate are shown in Fig. 2. The solid curve in the C-Si region was taken from Pief. 1 and demonstrates that acceptable agreement, can be obtained with the experimental $\tau_{s}$ for $\mathrm{c}-\mathrm{Si}$. The origin of the apparent displacement of the calculated curve from the experimentäl points at higher values of $E_{\ell}$ is not clear (however, note the experimental error bars). The short-dashed curve in the a-Si region was obtained from calculations with $\mathrm{T}_{\mathrm{a}}=1150^{\circ} \mathrm{C}, \mathrm{L}_{\mathrm{a}}=1320 \mathrm{~J} / \mathrm{g}$, and $\mathrm{K}_{\mathrm{a}}=0.02 \mathrm{~W} / \mathrm{cm}-\mathrm{K}$, while the long-dashed curve used the same values of $L_{a}$ and $K_{a}$ and $T_{a}=T_{c}=1410^{\circ} \mathrm{C}$. Both curves give reasonably good agreement with experiment, given the experimental error bars; thus, a choice between the two sets of parameters is not obvious. In fact, a series of calculations in which $T_{a}$ was gradually increased to $T_{C}$ simply gave a family of curves lying between the two shown. Since all of these pairs of $T_{a}, L_{a}$ values also give satisfactory agreement with the $t_{m}\left(E_{\ell}\right)$ curves of Fig. 1 , we again see that it is $K_{a}$, not $T_{a}$ or $L_{a}$, which strongly influences the results. The peak in the short-dashed curve at $0.5 \mathrm{~J} / \mathrm{cm}^{2}$ may be an artifact of the present modeling; however, both the calculations and TEM results show that this is approximately the value of $E_{\ell}$ for which the remaining a-Si layer has become so 
thin as to greatly increase heat flow to the c-Si substrate. Thus, reflectivity measurements and model calculations of surface melt duration are in satisfactory overall agreement for the 100-nm-thick a-Si layer, lending confidence that the model calculations can be used to infer both the depth of melting into a-Si and temperature profiles in the undercooled 1-Si, prior to solidification.

Typical results of calculations of temperature vs depth $[3,4]$ are shown in Fig. 3, while melt depth profiles vs time [4] are shown in Fig. 4, for a 100-nm-thick a-Si layer and a 532-nm wavelength, 18-nsec FWHM laser pulse. (The surface melt durations presented in Fig. 2 were obtained from calculations similar to those shown in Fig. 4.) In these calculations we again took $\mathrm{T}_{a}=1150^{\circ} \mathrm{C}$ and $\mathrm{L}_{a}=1320 \mathrm{~J} / \mathrm{gm}$ and $\mathrm{K}_{\mathrm{a}}=0.02$ $W / \mathrm{cm}-\mathrm{K}$, with other parameter values for $\ell-$ and $\mathrm{c}-\mathrm{Si}$ as described above. Figure 3 shows temperature profiles at the times when the melt front for each $E_{\ell}$ reaches its maximum penetration. $T_{n}$ was varied to obtain approximate agreement with the TEM results for the widths of the $F G, L G$, and a-Si regions; Fig. 3 shows $T_{n}=1210^{\circ} \mathrm{C}$ but this value depends on the assumed value of $T_{a}$. Figure 3 indicates that the temperature of the $\ell-S i$ for 0.2 $\mathrm{J} / \mathrm{cm}^{2}$ does not rise above $T_{n}$, so that only $F G p-S i$ is expected at the surface (in agreement with TEM), followed by the remainder of the a-Si. For $0.3 \mathrm{~J} / \mathrm{cm}^{2}, T>T_{n}$ in the region nearest the surface, and a thin region of $L G$ material is expected to grow from the underlying, bulk-nucleated $F G$ material. As $E_{\ell}$ increases, the $L G$ region grows in size while the $F G$ region shrinks until at $\sim 0.6 \mathrm{~J} / \mathrm{cm}^{2}$ only $\sim 20 \mathrm{~nm}$ of $F G \mathrm{p}-S_{i}$ remains; for $E_{\ell}$ $\geq 0.8 \mathrm{~J} / \mathrm{cm}^{2}$ the melt front penetrates completely through the $a-s i$. The 
kink in each of the curves in Fig. 4 at $\sim 100 \mathrm{~nm}$ occurs because $T_{a}<T_{C}$ and $L_{a}<L_{c}$; the melt front pauses until enough energy is absorbed for it to penetrate the $a-c$ interface. For $0.6 \leqslant E_{\ell} \leqslant 0.8 \mathrm{~J} / \mathrm{cm}^{2}$, the front contacts the interface but does not penetrate it. For $E_{\ell} \leqslant 0.6 \mathrm{~J} / \mathrm{cm}^{2}$, the temperature of part of all of the $\ell-S i$ is $\left\langle T_{n}\right.$ and nucleation occurs in an extended region for which no well-defined melt front exists, as indicated schematically by the cross hatching on Fig. 4.

Initial calculations for 400-nm-thick a-Si layers gave melt-front penetrations much less ihan the measured combined thickness of the FG and LG regions. This seemed to imply that bulk nucleation and grain growth, accompanied by the release of latent heat, occurred before the melt front reached its maximum penetration and increased the depth of melting. We might expect (and the calculations confirmed) this to be an important effect for thick layers but not for 100-nm-thick layers. Bulk nucleation during melt-in was simulated by allowing an increment $\Delta \mathrm{E}_{n}$ of latent heat to be released during melt-front penetration. $\Delta \mathrm{E}_{n}$ was estimated from the relationship $\Delta E_{n}=F_{\rho} L_{c} X_{I n}$, where $X_{m}$ is the measured maximum penetration at a given $E_{\ell}, \rho$ is the density, and $F$ is a factor interpreted as the fraction of $\ell-S i$ that solidifies before the melt front reaches $X_{m}$. To be physically meaningful, $F$ must be $\leq 1$ and we found $F \simeq 0.5-0.6$ for most $E_{\ell}$. Moreover, the temperature profiles when $x_{m}$ was reached again indicated that the widths of the LG and FG p-Si regions extracted from the calculations were in good agreement with the TEM values if $T_{n} \simeq 1210^{\circ} \mathrm{C}$. The model calculations clearly indicate that molten material can exist in the interior of, the sample after the surface has resolidified. 
The thsory of homogeneous bulk nucleation [19] yields an expression for the nucleation rate I of the form

$$
I=I_{0} \exp \left(-\Delta G^{*} / k T\right)
$$

$I_{0}$ is a factor having units of $\mathrm{cm}^{-3} \mathrm{~s}^{-1}$ and magnitude of approximately 1035. $\Delta G^{*}$ is the free energy of a critical nucleus at temperature $T$. For spherical nuclei of radius $r$ the free energy is given by $\Delta G=4 \pi r^{2} \sigma+$ $4 \pi r^{3} \Delta G_{V} / 3$, in which $\sigma$ is the surface free energy and $\Delta G_{V}$ the volume free energy. Setting $d \Delta G / d r=0$ gives

$$
r^{*}=-2 \sigma / \Delta G_{V}, n^{*}=4 \pi r * 3 N_{V} / 3
$$

and

$$
\Delta G^{*}=16 \pi \sigma^{3} / 3\left(\Delta G_{V}\right)^{2} .
$$

$r^{*}$ is the radius of the critical nucleus, $n^{*}$ is the number of atoms in it, $N_{V}$ is the number of atoms/unit volume. If the difference in the heat capacity $C_{p}$ between the liquid and the solid is neglected, $\Delta G_{V}$ can be expressed in terms of the undercooling $\Delta T \equiv T_{C}-T$ as $\Delta G_{V}=L_{C} \Delta T / T_{C}$.

If indeed the FG p-Si is due to bulk nucleation, $\sigma$ can be calculated as follows. TEM $[3,5]$ indicated an average grain size of $\sim 10 \mathrm{~nm}$ or a volume of $10^{-18} \mathrm{~cm}^{3}$. Knowing the thickness $x_{p}$ of the FG $p-S i$ region from TE:M and an approximate value of its formation time $t_{p}$ from the model calculations, I can be determined under the assumption that each $10-\mathrm{nm}$ grain resulted from a single nucleation event. For example, from the results leading to Figs. 3 and 4 for $E_{\ell} \simeq 0.3 \mathrm{~J} / \mathrm{cm}^{2}$, we estimate $x_{p} \simeq 50$ $\mathrm{nm}, t_{\mathrm{p}} \simeq 10 \mathrm{~ns}$, and find $\mathrm{I} \simeq 10^{26} \mathrm{~cm}^{-3} \mathrm{~s}^{-1}$. With a value of I established, Eqs. (1) 'and (2) and the expression for $\Delta G_{V}$ can be used to relate 
$\sigma$ to $\Delta T$. Following Ref. 8 , we took $\Delta T=265 \mathrm{~K}$ and calculated $\sigma, r^{*}$, and $n^{*}$ with the results: $\sigma=219 \mathrm{erg} / \mathrm{cm}^{2}, r^{\star}=0.66 \mathrm{~nm}$, and $n^{\star}=60$ atom. For the lower limit of $\Delta T(=390 \mathrm{~K})$ estimated in Ref. 8 , we found $\sigma=275$ $\mathrm{erg} / \mathrm{cm}^{2}, r^{\star}=0.57 \mathrm{~nm}$, and $n^{\star}=39$ atom. Having fixed $\sigma, \Delta T$ can be varied to determine its effects on $I, R^{*}$, and $n^{*}$, with typical results shown in Table I. We note that the values of I in Table I are much greater than those estimated by Turnbul1 [20] but are not inconsistent with values found from molecular dynamics calculations [21].

We will summarize our view of the physical processes that take place. The 18-ns laser pulse causes the a-Si to melt at a $T \sim 300 \mathrm{~K}$ less than $T_{C}$. Melting is either incomplete, in the sense that small clusters of atoms remain in the melt, or nucleation occurs so rapidly that the two situations are nearly indistinguishable on the time scale of the present experiments. In either case, a fraction of the latent heat is, in effect, released while the melt front is still advancing; this release is essential for forcing the melt front to its maximum penetration. In the FG $\mathrm{p}-\mathrm{S} i$ region the temperature of the $\ell-S i$ is $\leq T_{n}$ long enough for nucleation to occur. Because nucleation will occur in those regions first melted, the surface may solidify before tise deeper-lying regions. We have not yet determined the extent of the "slush zone" in which $2-S i$ and $c-S i$ coexist at any particular time, but we note that the physical properties of such a mixture are likely to be quite different from those of either pure $\ell$-Si or c-Si. Finally, we observe that $T_{n}$ should appear on the free-energy diagram, as in Fig. 5. From this diagram [4] it can be seen that slow cooling of $\ell-S i$ from $T_{C}$ will result in nucleation of $\mathrm{p}-\mathrm{Si}$ rather than formation of a-Si. Therefore, the $\ell \rightarrow$ a transition that can be produced 
using picosecond or ultraviolet nanosecond laser pulses must occur either because the system is undercooled to $T_{\mathrm{a}}$ so quickly that nucleation cannot occur or because a large number of mistakes are made in the incorporation of atoms into the growing interface at high solidification velocities. (To the extent that the free-energy diagram of Fig. 5 can be used, the latter situation corresponds roughly to a path from the e-Si line to, and along, the superheated $a-S i$ line at temperatures above $T_{n} \cdot$ ) Thus, we see that the a-Si phase must be "trapped" by nonequilibrium thermodynamic and kinetic effects [23].

Acknowledgments

The authors thank F. W. Young; J.r. and G. E. Jellison, Jr. for several informative discussions. This research was sponsored by the Division of Materials Sciences, U.S. Department of Energy under Contract No. DE-AC05-840R21400 with Martin Marietta Energy Systems, Inc. 


\section{References}

[1] D. H. Lowndes, R. F. Wood, and R. D. Westbrook, App1. Phys. Lett. 43, $258(1983)$.

[2] D. H. Lowndes, R. F. Wood, and J. Narayan, Phys. Rev. Lett. 52, 561 (1984).

[3] D. H. Lowndes, R. F. Wood, and J. Narayan, Mat. Res. Soc. Symp. 23, 99 (1984).

[4] R. F. Wood, D. H. Lovindes, and J. Narayan, App 1. Phys. Lett. 44, 770 (1984).

[5] J. Narayan and C. W. White, Appl. Phys. Lett. 44,35 (1984).

[6] G. L. 01son, S. A. Kokorowski, J. A. Roth, and L. D. Hess, Mat. Res. Soc. Symp. 13, 141 (1983) and references herein to earlier papers by these authors.

[7] G. L. 0lson, J. A. Roth, L. D. Hess, and J. Narayan, "Proc. of U.S.-Japan Seminar on Solid Phase Epitaxy and Interface Kinetics," 0iso, Japan (June 20-24, 1983).

[8] E. P. Donovan, F. Spaepen, D. Turnbu11, J. M. Poate, and D. C. Jacobson, App1. Phys. Lett. $\underline{42}, 698$ (1983).

[9] B. G. Bagley and H. S. Chen, in AIP Conf. Proc. 50, 9i (1979).

[10] F. Spaepen and D. Turnbu11, ibid., p. 73.

[11] P. Baeri, G. Foti, J. M. Poate, and A. G. Cullis, Phys. Rev. Lett. $\underline{45}, 2036(1980)$.

[12] J. A. Knapp and S. T. Picraux, App1. Phys. Lett. 38, 873 (1981). 
[13] See, e.g., P. L. Liu, R. Yen, N. Bloembergen, and R. T. Hodgson, App1. Phys. Lett. 34, 864 (1979); R. Tsu, R. T. Hodgson, T. Y. Tan, and J. E. Bagl in, Phys. Rev. Lett. 42, 1356 (1979); A. G. Cullis, H. C. Weber, N. G. Chew, J. M. Poate, and P. Baeri, Phys. Rev. Lett. 49 , $219(1982)$.

[14] G. E. Jellison, Jr. (private communication).

[15] C. J. Glassbrenner and G. A. Slack, Phys. Rev. A 134, 1058 (1964).

[16] H. J. Goldsmid, M. M. Kaila, and G. L. Pa'ıl, Phys. Status Solidi A 76, K31 (1983).

[17] H. C. Webber, A. G. Cullis, and N. G. Chew, Appl. Phys. Lett. 43 , 669 (1983).

[18] R. F. Wood and G. E. Giles, Phys. Rev. B 23, 2923 (1981).

[19] R. F. Wood, to be published; see J. H. Holloman and D. Turnbull, Progr. Metal Phys. IV, 333 (1953).

[20] D. Turnbu11, J. Appl. Phys. 21, 1022 (1950).

[21] C. S. Hus and A. Rahman, J. Chem. Phys. 70, 5234 (1979).

[22] The possibility that a-Si can be substantially superheated has been suggested previously; see, e.g., Ref. 8.

[23] R. F. Wood, Mat. Res. Soc. Symp. 13, 83 (1983). 
Table I. I, $r^{*}$, and $n^{*}$ vs $\Delta T / T_{C}$ for $\sigma=275 \mathrm{erg} / \mathrm{cm}^{2}$.

\begin{tabular}{ccccc}
$\Delta \mathrm{T} / \mathrm{T}_{\mathrm{c}}$ & $\begin{array}{c}\mathrm{T} \\
(\mathrm{K})\end{array}$ & $\left(\mathrm{cm}^{-3} \mathrm{~s}^{-1}\right)$ & $\begin{array}{c}\mathrm{r}^{*} \\
\mathrm{~nm})\end{array}$ & $\mathrm{n}^{*}$ \\
\hline 0.13 & 1464 & $7.9 \times 10^{6}$ & 1.01 & 214 \\
0.15 & 1431 & $5.0 \times 10^{15}$ & 0.87 & 139 \\
0.17 & 1397 & $4.1 \times 10^{19}$ & 0.77 & 96 \\
0.19 & 1363 & $2.4 \times 10^{22}$ & 0.69 & 69 \\
0.21 & 1330 & $2.6 \times 10^{24}$ & 0.62 & 51 \\
0.23 & 1296 & $8.7 \times 10^{25}$ & 0.57 & 39 \\
0.25 & 1262 & $-1.3 \times 10^{27}$ & 0.52 & 30 \\
\hline \hline
\end{tabular}




\section{Figure Captions}

Fig. 1. Measured time of onset of melting $\left(t_{m}\right)$ for a-Si (circles) and for $\mathrm{C}-\mathrm{S} j$ (dots) vs $\mathrm{E}_{\ell}$. Also shown are the results of mocel calculations of $t_{m}$ for $c-$ and a-Si (see text).

Fig. 2. Measured duration of the high-reflectivity phase (surface melt duration, $\tau_{s}$ ) for a-Si (circles) and for c-Si (dots) vs $E_{l}$. The error bars are derived from the measured rms precision of the $E_{\ell}$ calibrations. Also shown are the results of model calculations of $\tau_{s}$ for a-Si (see text).

Fig. 3. Profiles of temperature vs depth in undercooled liquid silicon. Each profile is shown at the time of maximum melt-front penetration for the given $E_{\ell}$.

Fig. 4. Melt-front profiles vs time for different laser $E_{\ell}$. The crosshatching indicates the region in which TEM indicates that a welldefined melt front does not exist (see text).

Fig. 5. Schematic representation of Gibbs free energy vs temperature for a-Si and $\ell-S i$ relative to $c-S i$. 


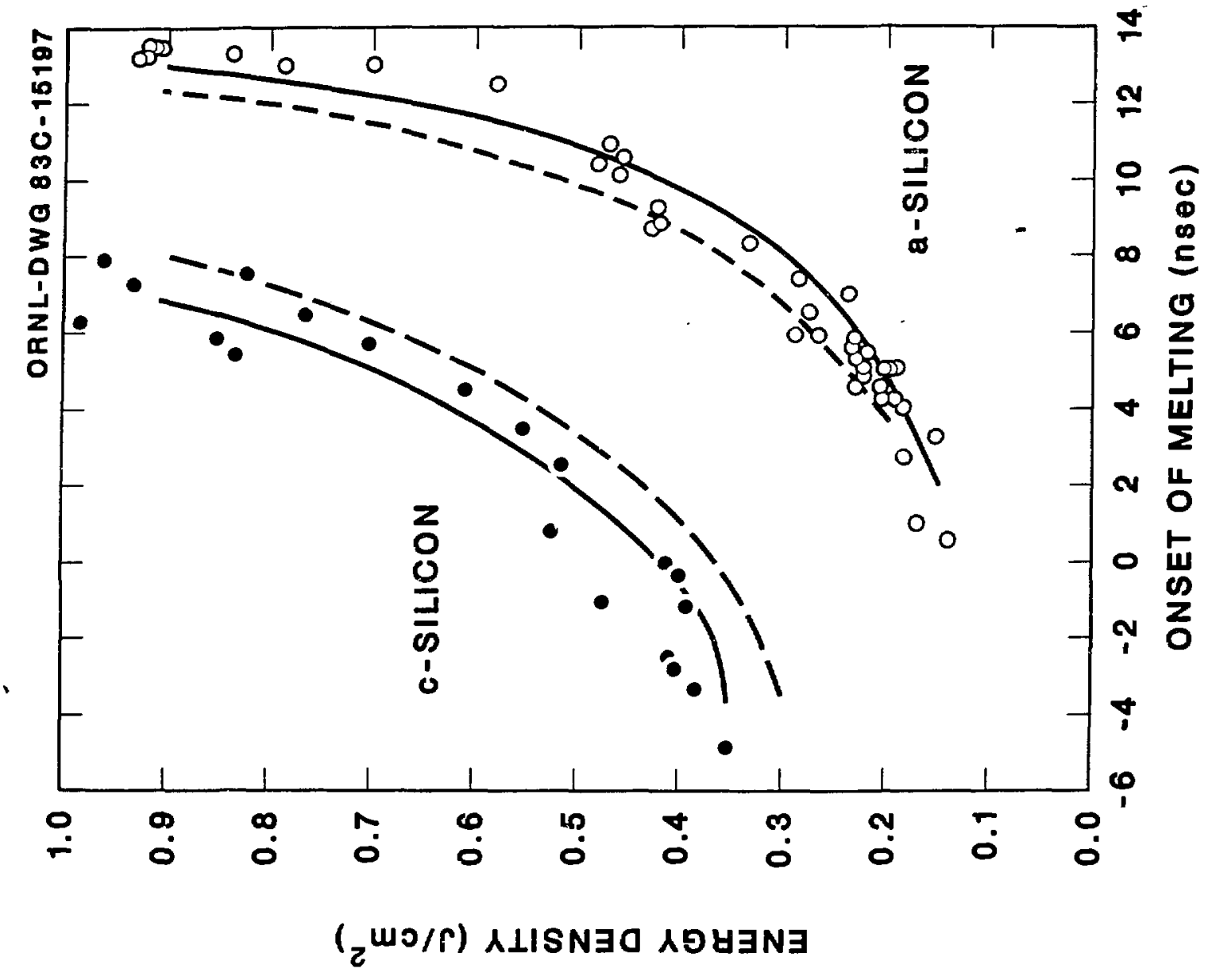




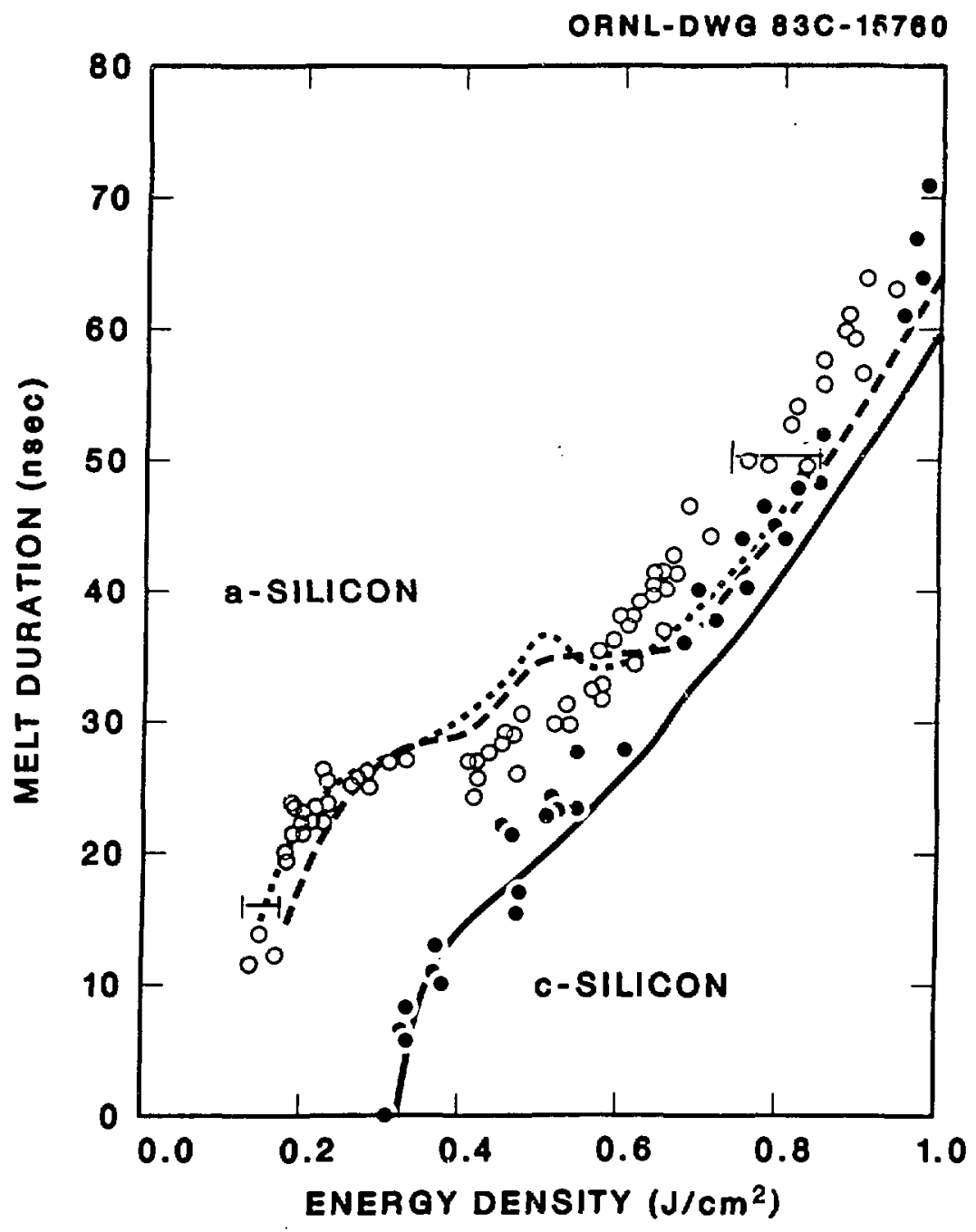




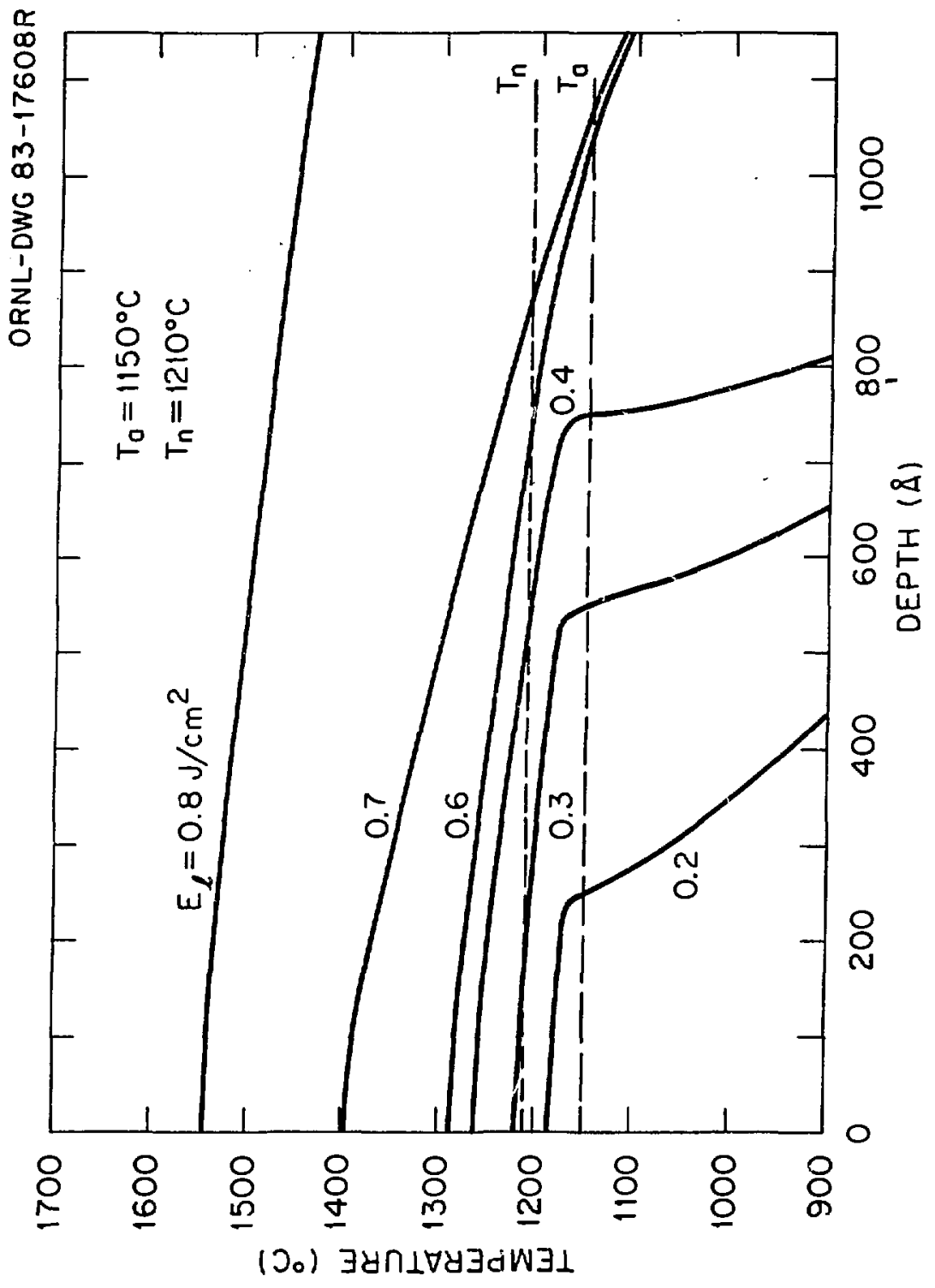




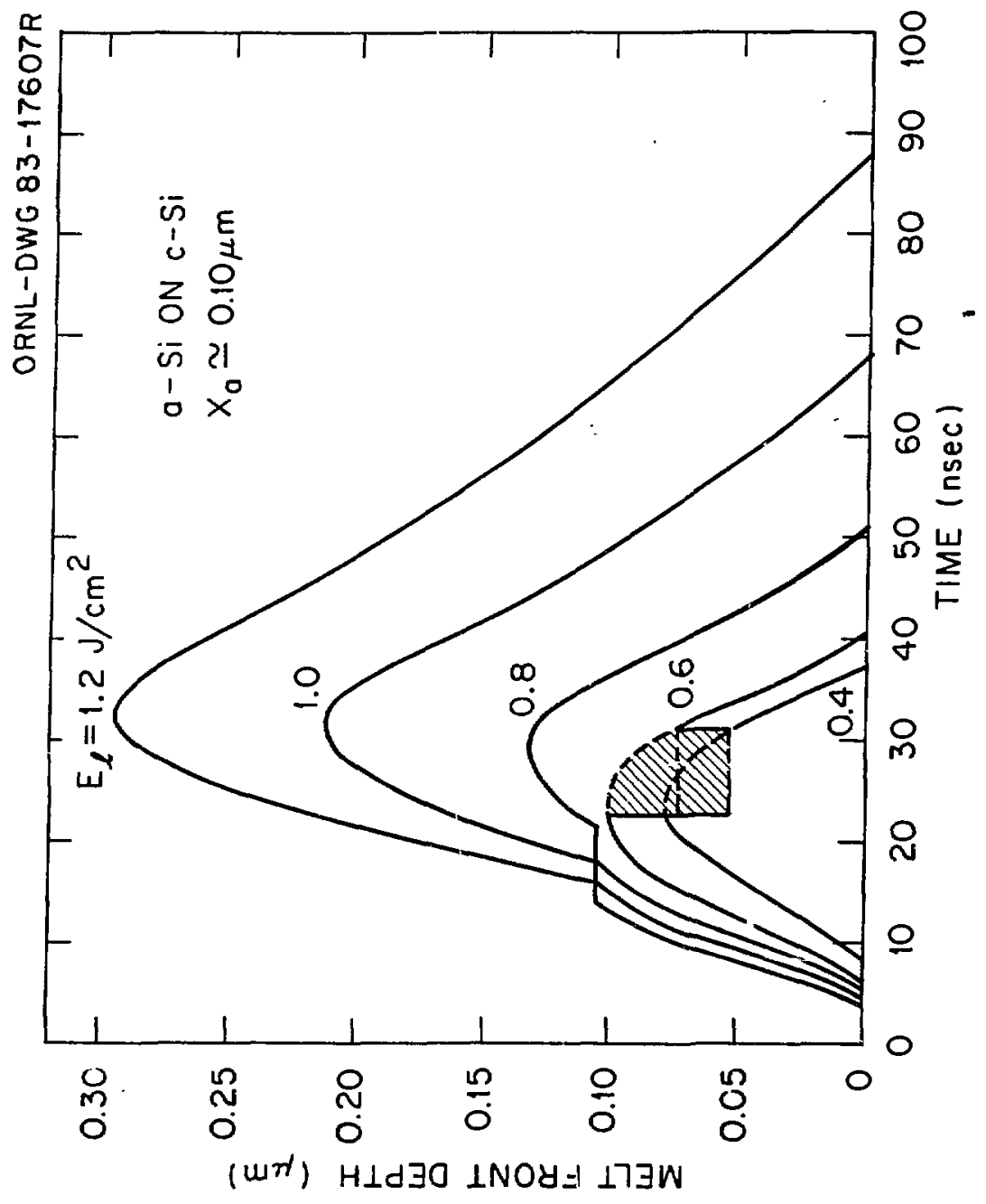


ORNL-DWG 83.17672R

RELATIVE FREE ENERGIES OF a-, c- AND l-PHASES

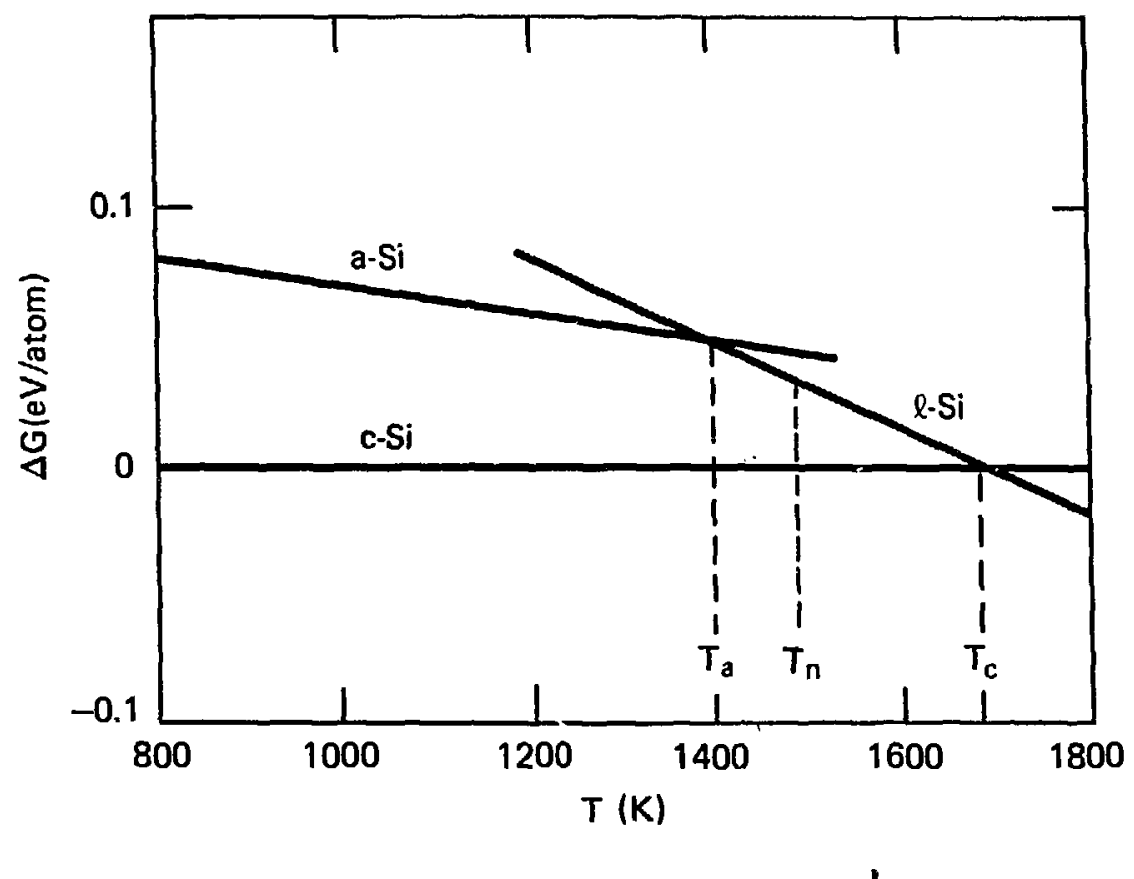

